\title{
Advanced Train and Traffic Control Based on Prediction of Train Movement*
}

\author{
Shigeto HIRAGURI**, Yuji HIRAO**, Ikuo WATANABE**, Norio TOMII*** and Shinichi HASE***
}

\begin{abstract}
Trains are often forced to decelerate or stop between stations on commuter lines due to the delay of the preceding train. If a train stops between stations, both the travel time and the interval between trains will increase. This situation has an adverse effect on energy consumption. To solve this problem, we propose a new train control method based on the prediction of train movement and data communications between railway sub-systems. Computer simulations are carried out to verify the effect of the proposed method. As a result, it has been proved that the new method reduces the train stopping time between stations and the electric energy consumption at substations.
\end{abstract}

Key Words: Railway, Adaptive Control, Train Control, Traffic Control, Prediction, Communications, Energy Consumption

\section{Introduction}

There are very large transport demands for commuter lines in Japan, where trains are operated at very high frequencies. In metropolitan areas, for example, the train headway is about two or three minutes in the morning. Furthermore, stations are routinely crowded with passengers, and trains often dwell for longer times than scheduled. In this situation, trains approaching a station are often forced to decelerate or stop between stations. This phenomenon easily occurs especially where the train headway makes a bottleneck. Once a train stops between stations, the running time of the train increases and recovery from traffic disruption delays ${ }^{(1)}$. From the viewpoint of energy consumption, the occurrence of this phenomenon is also undesirable because trains may repeat unexpected acceleration and deceleration.

One of the train control methods to solve the problem

* Received 13th January, 2004 (No. 04-4021)

** Signalling and Telecommunications Technology Division, Railway Technical Research Institute, 28-38 Hikari-cho, Kokubunji-shi, Tokyo 185-8540, Japan. E-mail: hiraguri@rtri.or.jp, hirao@rtri.or.jp, ikuo@rtri.or.jp

*** Transport Information Technology Division, Railway Technical Research Institute, 2-8-38 Hikari-cho, Kokubunji-shi, Tokyo 185-8540, Japan. E-mail: tomii@rtri.or.jp

${ }^{* * * *}$ Power Supply Technology Division, Railway Technical Research Institute, 2-8-38 Hikari-cho, Kokubunji-shi, Tokyo 185-8540, Japan. E-mail: hase@ rtri.or.jp is to avoid unnecessary stops with the headway kept as short as possible ${ }^{(2)}$. To realise this control, detailed information on the running status of the preceding train is necessary. In the conventional railway system, on the other hand, the information given to trains to control its speed is only signal aspects. Therefore, it is difficult to control trains to run at the optimal speed adapted to the traffic status.

We are now studying a new train and traffic control system with which railway sub-systems such as rolling stock, electric power supply system, traffic control system and signalling system, co-operate based on the information about train movement and traffic ${ }^{(3)}$. In this paper, we describe the train control method which is a core function of the new system. The control method named "prediction control" is based on the information of predicted time when the train departs from a station. To evaluate the effects of proposed control method, we carried out computer simulations of traffic and an electrical feeding circuit. This paper reports the results of the simulation.

\section{Prediction Control}

\subsection{Outline of control method}

Trains normally run according to a time table and a basic position-speed curve produced beforehand. When the departure of a train from a station delays, the train approaching the station is forced to implement unexpected deceleration or a stop according to the given signal aspect. One of the purposes of the prediction control is to realise train control method which is dynamically adapted 
to traffic status, reduce augmentation of the influence of delay and expedite recovery from traffic disruption as soon as possible.

The basic preconditions for the prediction control are the existence of a data transmission means between trains and the ground, and continuous detection of train position. In this paper, we assume that "Digital ATC"(4) as the signalling system, which is based on digital data transmission to use track circuits. (Note: In Japan, automatic train control (ATC) is a cab signalling system and applies or releases brake automatically by supervising their speed.) In the conventional ATC, only discrete speed signals of each track circuit are transmitted to trains. On the lines where Digital ATC is installed, trains detect their position continuously and control their speed according to the continuous braking speed curve which is generated from the number of unoccupied track circuits.

The basic concept of the prediction control is as follows.

(1) The control information which consists of time " $t_{c}$ ", position " $p_{c}$ " and speed " $v_{c}$ " are given to trains.

( 2 ) Trains control their speed to reach " $p_{c}$ " and " $v_{c}$ " at " $t c$ ".

There are two kinds of control information described in (1). The first is the information about the arrival at the next station and the second is the information for avoiding a stop between stations. Trains select the appropriate information according to the traffic status and set it as the target for speed control. Typical contents of the first information are as follows. The time is the arrival time at the next station specified by the time table; the position is the prescribed stopping position at the station, and the speed is equal to zero. When the system is advanced further, the information of the time may dynamically be decided according to the traffic status by the traffic control system. The contents of the second information are as follows. The time is the "predicted time" that indicates when the braking speed curve to stop before the home route is cleared. The position and the speed are the "approach point" on the braking speed curve. If a train selects the second information as the target, it controls speed to reach the approach point at the predicted time. In the next section, the train control method based on the second information is described.

\subsection{Control method to avoid a stop between stations}

The train headway normally makes a bottleneck at the home route. When traffic is disrupted and the interval with the preceding train becomes short, the train approaching a station often decelerates or stops before the home route according to the braking speed curve. In this case, if the time when the braking speed curve is cleared is known beforehand, the train is able to avoid unexpected deceleration or a stop by running at lower speed than the normal speed. On the other hand, the train is required to keep the headway as short as possible by approaching the preceding train to reduce its influence on the following trains. In order to realise this speed control, the train is required to reach a certain point on the braking speed curve at the time when the curve is cleared.

The concept of this control method is shown in Figs. 1 and 2. The predicted time is calculated from the predicted time when the preceding train leaves the station, and it is transmitted to the following train approaching the station. The following train controls its speed to avoid an unexpected stop between stations by reaching the approach point at the predicted time.

The method to determine the approach point is as follows. It is assumed that the train runs along the defined position-speed curve from the approach point to the prescribed stopping position at the station. In this paper, we assume the shape of that curve as shown in Fig. 2. In this case, the running time " $t$ " from the clearing of braking speed curve to the train arrival at the station is expressed by the Eq. (1). The speed " $v_{c}$ " which minimises " $t$ " is expressed by the Eq. (2), and this value is the speed at the approach point. The position of the approach point is derived from the value of " $v_{c}$ " and the deceleration of the braking speed curve.

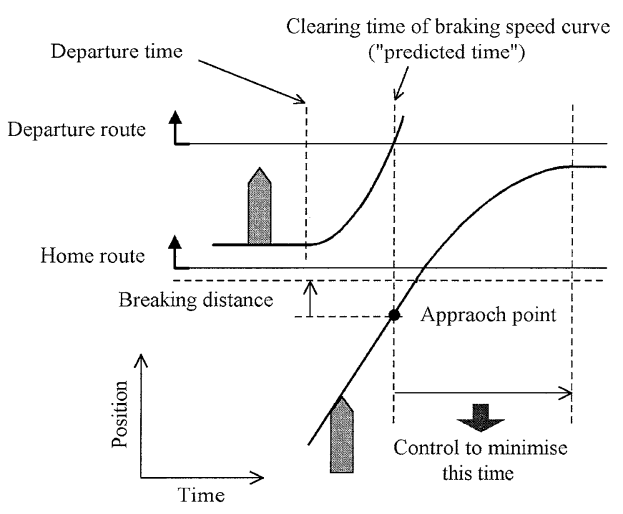

Fig. 1 Concept of prediction control

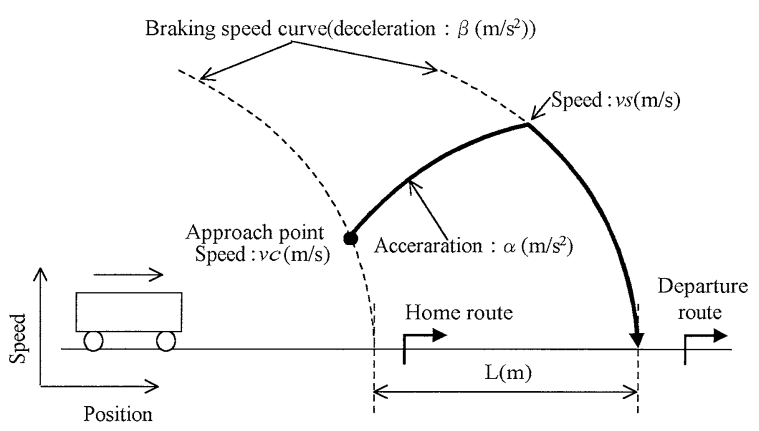

Fig. 2 Approach point and position-speed curve 


$$
\begin{aligned}
& \left\{\begin{array}{l}
t=\frac{v_{s}-v_{c}}{\alpha}+\frac{v_{s}}{\beta} \quad[\mathrm{s}] \\
v_{s}=\sqrt{v_{c}^{2}+\frac{2 \alpha \beta L}{\alpha+\beta}} \quad[\mathrm{m} / \mathrm{s}]
\end{array}\right. \\
& v_{c}=\sqrt{\frac{2 \beta^{3} L}{(\alpha+\beta)(\alpha+2 \beta)}} \quad[\mathrm{m} / \mathrm{s}]
\end{aligned}
$$

$\alpha\left[\mathrm{m} / \mathrm{s}^{2}\right]$ : Acceleration of a train.

$\beta\left[\mathrm{m} / \mathrm{s}^{2}\right]$ : Deceleration of a train.

$v_{c}[\mathrm{~m} / \mathrm{s}]$ : Train speed when the braking speed curve to stop before the home route is cleared.

$v_{s}[\mathrm{~m} / \mathrm{s}]$ : Train speed when it reaches the braking speed curve to stop at the prescribed position at the station.

$L[\mathrm{~m}]$ : Distance from stopping position before the home route to the stopping point at the station.

It is possible to determine the approach point from the performance of rolling stock and characteristics of the line beforehand. Therefore, among the elements of control information, only the predicted time changes dynamically according to the traffic status.

\subsection{Control method adaptive to traffic situation}

As described above, the train sets appropriate control information as the target, and controls its speed according to it. The sequence and method of speed control are as follows, which are also described in Fig. 3.

(1) The train generates the position-speed curve to arrive at the next station at the earliest time.

(2) The train compares the arrival time at the next station " $T_{s}$ " specified by the time table with the arrival time " $T_{f}$ " when the train runs along the position-speed curve generated in (1).

(2)-(a): $\quad T_{s} \geqq T_{f}$

The train generates the position-speed curve to arrive at the next station at " $T_{s}$ " as a substitution of the curve generated in (1), and makes the new curve the basis of the control described in (3).

$$
\text { (2)-(b): } \quad T_{s}<T_{f}
$$

The train makes the curve generated in (1) the basis of the control described in (3).

(3) The train calculates whether it should decelerate according to the braking speed curve to stop before the home route when it runs along the curve which is adopted as the basis in (2).

(3)-(a): The train does not decelerate.

The train controls its speed to arrive at the next station at the target time. The target time is " $T_{s}$ " if the positionspeed curve is substituted in (2), or " $T_{f}$ " if the curve is not substituted.

(3)-(b): The train decelerates.

The train controls its speed to reach the approach point at the predicted time. In this case, the target time is the predicted time.

\subsection{Speed control method}

There are various position-speed curves to reach the target point (station or approach point) at the target time, but the train has to determine its speed by some means. A simple method is to calculate the control speed as shown in Fig. 4. In this method, it is assumed that the train runs along a constant target speed until it reaches the braking speed curve. However, the train is forced to frequently change the running mode which consists of acceleration, coasting and deceleration. On the other hand, from the

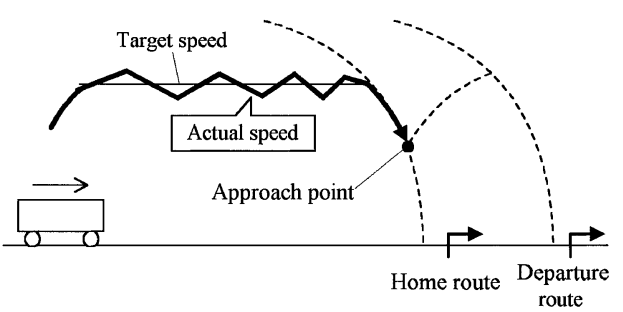

Fig. 4 Train control based on a constant target speed

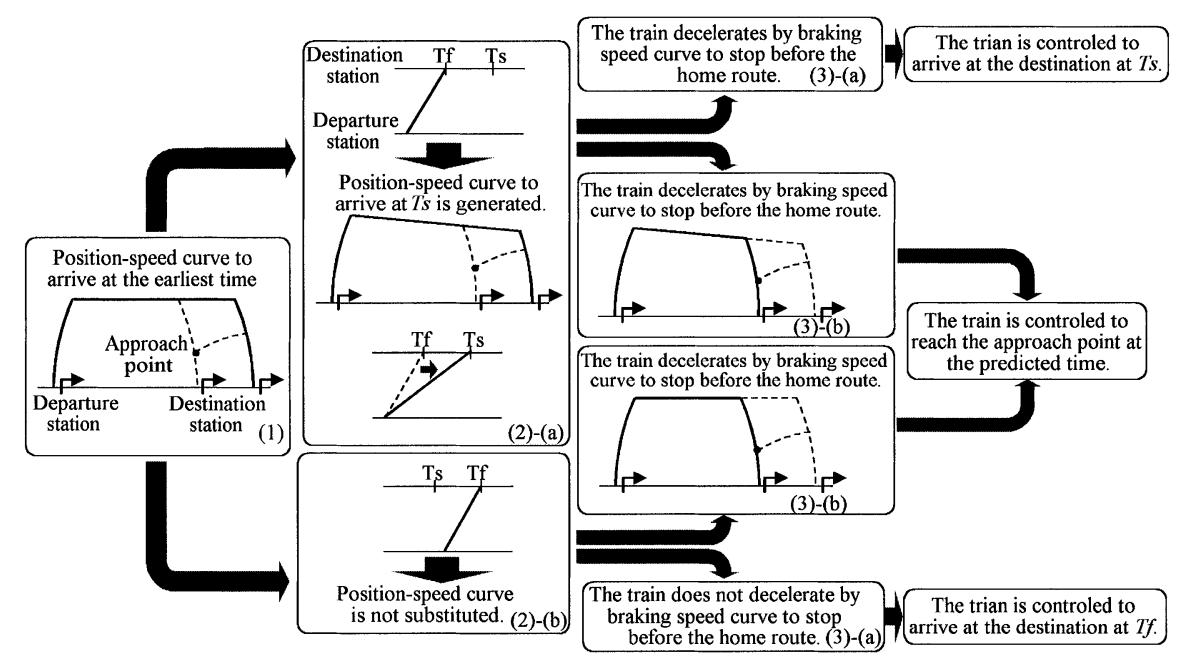

Fig. 3 Sequence of speed control 
viewpoint of saving energy, it is clarified that the train has to coast as long as possible after running at the maximum acceleration $^{(5)}$. Therefore, in the proposed method, the generation of position-speed curve described in the section 2.3 and the speed control are based on this concept. As shown in Fig. 5, while the train accelerates, it calculates the arrival time at the target point at every processing cycle on the assumption that it shifts to coasting. If the calculated time is equal to the target time, the train actually shifts from acceleration to coasting.

These processes are carried out every second. If the target time changes or an error occurs, the train corrects the target time or the error.

The train controls its speed and calculates the arrival time at the next station. On high traffic density lines, several trains may exist between stations. This requires to control trains other than the train approaching the station.

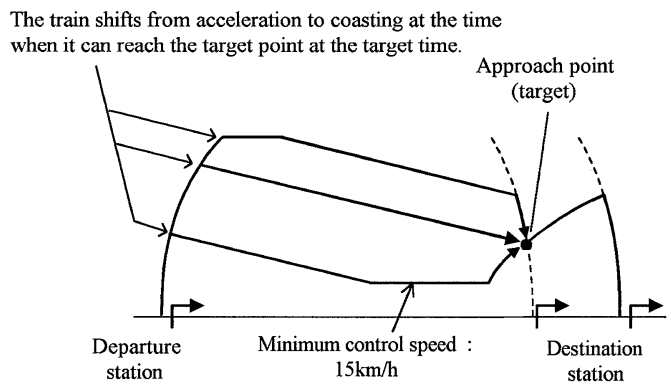

Fig. 5 Basic concept of speed control

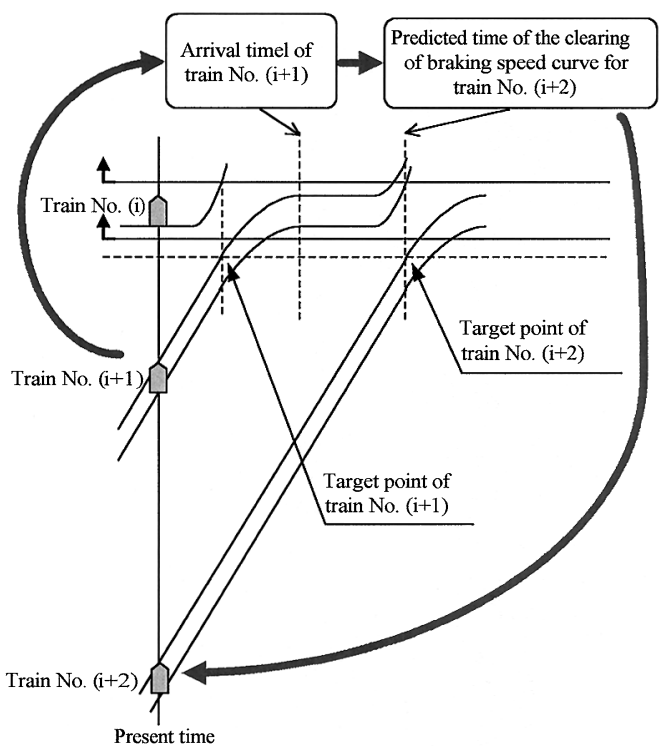

Fig. 6 Transmission of control information
Therefore, as shown in Fig. 6, the clearing time of braking speed curve for each train is predicted from the arrival time at the station which is calculated by the preceding train. By applying this process sequentially to each train existing between stations, all trains are able to control their speed based on the prediction control.

\section{Verification of the Proposed Method}

\subsection{Outline of verification}

The proposed method was verified by computer simulations because the system is complex with a large scale. We evaluate the movement of trains and performance of recovery from traffic disruption. Furthermore, a simulation of electrical feeding circuit was carried out in order to evaluate the effect of saving energy.

The computer program consists of a traffic simulation module and an electrical feeding circuit simulation module which interact with each other. For example, the program works to reflect changes in the performance of rolling stock according to the changes in the voltage supplied to each train.

\subsection{Conditions of simulation}

Figure 7 shows the location of stations and substations of the model line. The cycle of the simulations is equivalent to actual 0.1 seconds. Other conditions of the simulations are as follows.

( 1 ) Rolling stock
( a ) Control method: VVVF inverter control
(b ) Maximum speed: $33.3 \mathrm{~m} / \mathrm{s}\{120 \mathrm{~km} / \mathrm{h}\}$
(c ) Acceleration: $0.75 \mathrm{~m} / \mathrm{s}^{2}\{2.7 \mathrm{~km} / \mathrm{h} / \mathrm{s}\}$ (constant torque area)
(d ) Deceleration: $0.56 \mathrm{~m} / \mathrm{s}^{2}\{2.0 \mathrm{~km} / \mathrm{h} / \mathrm{s}\}$
(e ) Train length: $200 \mathrm{~m}$
(f) Train weight: $466 \times 10^{3} \mathrm{~kg}$ (passenger load factor $250 \%$ )

(2) Substation and feeding circuit

(a ) Capacity: $4500 \mathrm{~kW}$ (SS1), $9000 \mathrm{~kW}$ (SS2-SS9): SS means electric substation.

(b) Voltage regulation at the rated load: $8 \%$

(c ) Constants of the line Overhead contact line: $0.0215 \Omega / \mathrm{km}$ Rail: $0.014 \Omega / \mathrm{km}$

(3) Train schedule

(a ) A train departs every $135 \mathrm{~s}$ from the station $\mathrm{O}$ (minimum headway: $131.5 \mathrm{~s}$ )

(b ) Dwell time at stations: $30 \mathrm{~s}$ (stations B, C,

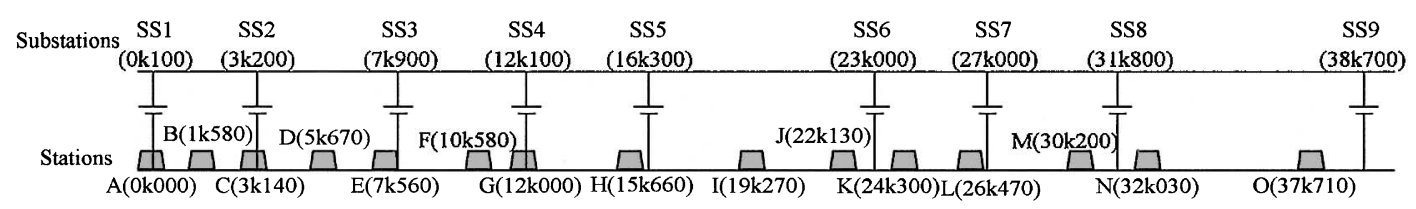

Fig. 7 Location of stations and substations of model line 
D, E, F, G, J, K, L, M and N), 40 s (stations $\mathrm{H}$ and $\mathrm{L}$ ), $50 \mathrm{~s}$ (station $\mathrm{I}$ )

(4) Factor of traffic disruption

The train delays 10 minutes at the station I.

\subsection{Results of simulation}

The simulated time is equivalent to about two hours. It was assumed that the delay of the train occurred at the station I. Prediction control was carried out only at the station I. The results of the simulations are as follows.

The resultant running paths when the prediction control is, and is not, carried out are shown in Figs. 8 and 9, respectively. In the case of Fig. 8, only several trains stop between the stations $\mathrm{J}$ and $\mathrm{I}$ just after the occurrence of delay, and most trains do not. On the other hand, in the case of Fig. 9, most trains stop repeatedly between sta-

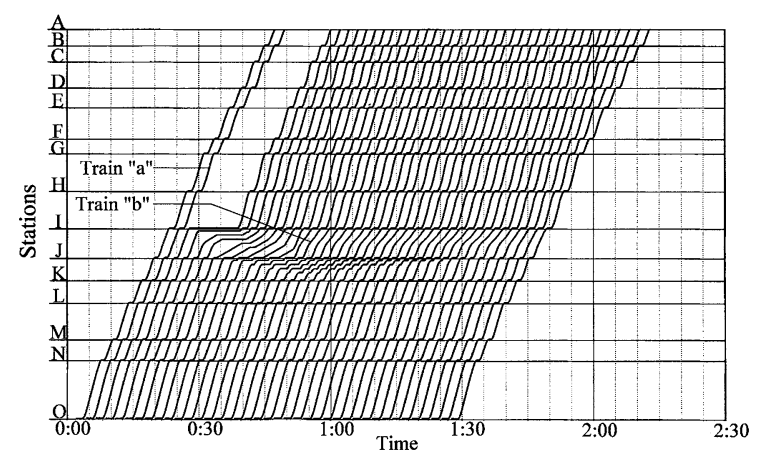

Fig. 8 Resultant running paths (with prediction control)

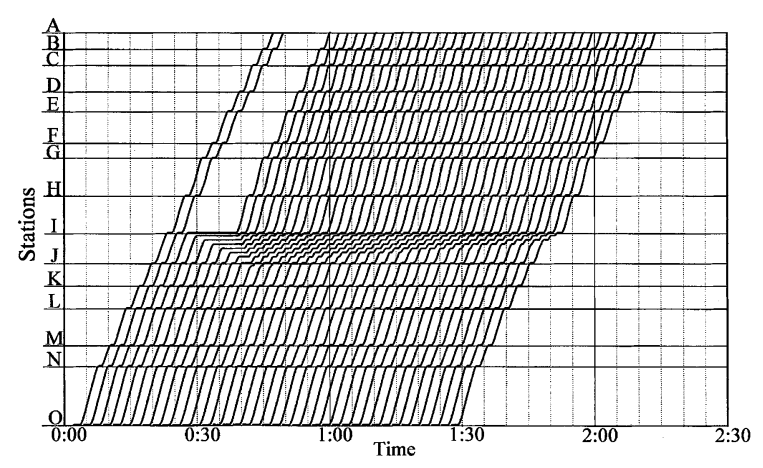

Fig. 9 Resultant running paths (without prediction control)

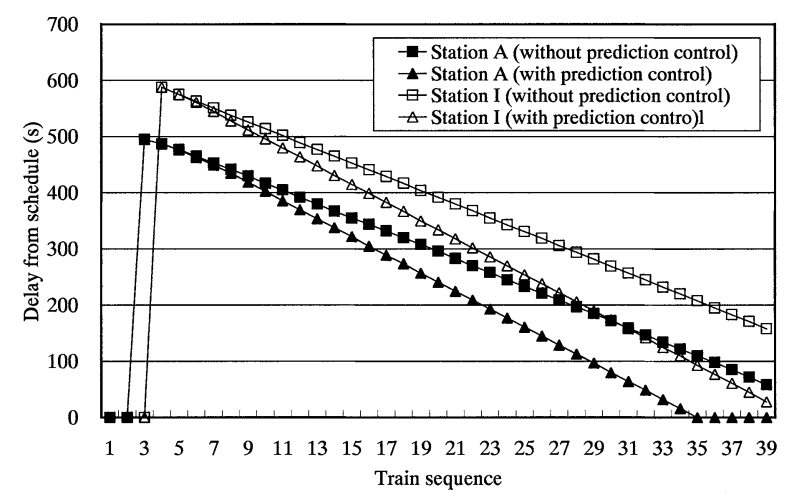

Fig. 10 Delay of arrival time tions $\mathrm{J}$ and $\mathrm{I}$, and even the last train stops before the station I. In Fig. 8, trains stop between stations $\mathrm{K}$ and J. The reason of this phenomenon is that in this verification, the prediction control was applied only to the station I. It will be solved by applying the prediction control to other stations.

Figure 10 shows the transition of the delay of the arrival time to that in the time table at stations A and I. The delay time decreases with the progress of the time. In the case where prediction control is carried out, the delay time decreases much faster, and the performance of recovery from traffic disruption is better. Furthermore, the delay time at the station $\mathrm{A}$ is shorter than that at the station I, because trains run faster by recovery operation than under the normal condition between the stations I and A.

Figures 11 and 12 show the resultant position-speed curve of the train which runs before and after delay occurs, respectively. In the case of Fig. 12, the train controls its

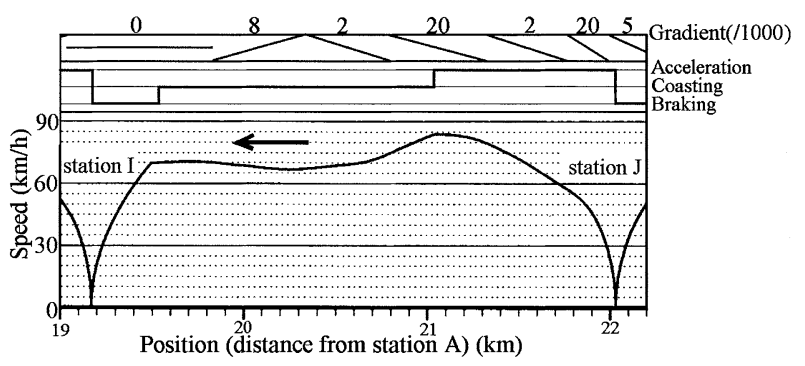

Fig. 11 Resultant position-speed curve (train "a" indicated in Fig. 8)

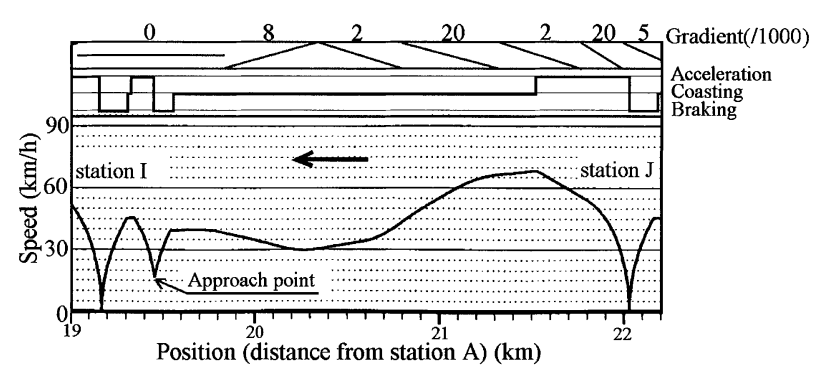

Fig. 12 Resultant position-speed curve (train "b" indicated in Fig. 8)

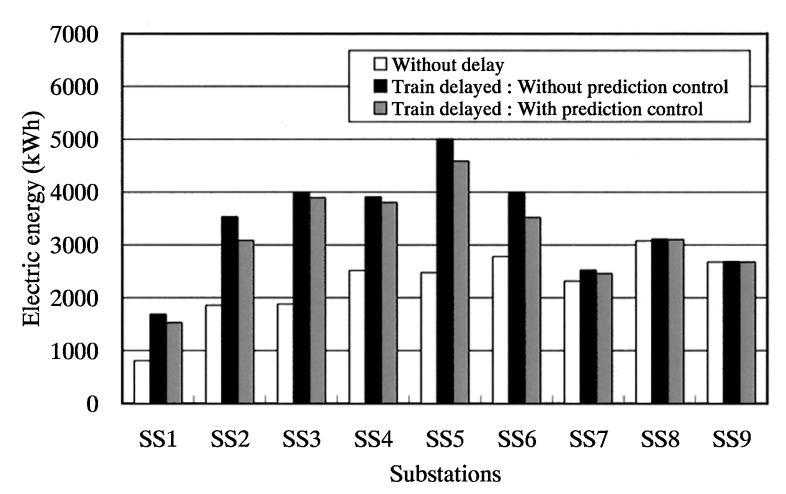

Fig. 13 Consumed electric energy at substations 


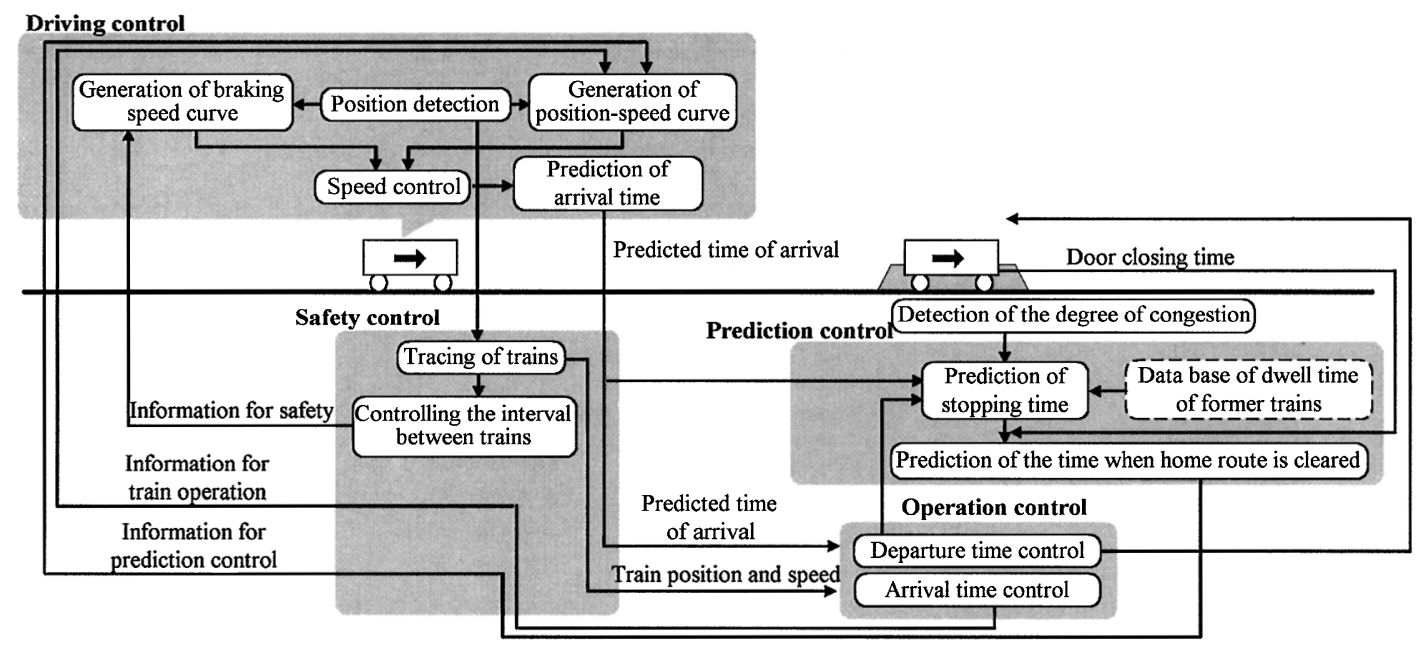

Fig. 14 Functional architecture of the new train control system

speed to reach the approach point at the predicted time, because the interval with the preceding train is shorter than that under the normal conditions. In both cases, trains continue coasting for a long time and the frequency of running mode shifts is small.

As shown above, the train controls its speed adapted to the traffic status by prediction control.

The consumed electric energy at each substation is shown in Fig. 13. In the case where the prediction control is carried out, the consumed energy at SS5 and SS6 which is nearest the station I is about $90 \%$ in comparison with the case where the control is not applied, and the total consumed energy at all substations is about $94 \%$.

\section{Function of Future System}

Figure 14 shows the functional architecture of the new train control system based on the prediction control.

In order to realise the prediction control, the function of digital data communications between trains and the ground and the function of on-board continuous position detection are required. Therefore, the Computer and Radio Aided Train control system $\left(\right.$ CARAT) ${ }^{(6)}$ or Digital ATC outlined in the section 2.1 makes the basis of the new system. The main features of CARAT are to use radio communications and realise a moving block system. The time of departure of a train from a station would be predicted from the time of arrival transmitted from the train approaching the station and the record of the past dwell time, etc. The train control would be a method such as the one for automatic train driving control because high precision speed control is required.

\section{Conclusion}

It is confirmed that the prediction control has excellent performance for recovery from traffic disruption.
Furthermore, the introduction of the control is effective for energy saving. The running state of trains has various states according to the gradient of line, speed restriction and conditions of signalling system, etc. We are aiming at establishing a detailed control logic through simulations to reflect various conditions.

In this paper, we have assumed that the dwell time at stations is given precisely. However, we recognise that it is required to evaluate the influence of error occurrence in prediction and establishment of a control logic based on the occurrence of errors. Furthermore, we will study the method of prediction of the dwell time at stations by referring to the previous result.

\section{References}

( 1 ) Fujiwara, M. and Sone, S., A Study on Short Headway Operation Based on Prediction of Departure Time, (in Japanese), Proceedings of IEEJ Transportation and Electric Railway Conference, TER-93-6, (1993), pp.49-58.

( 2 ) Sasaki, T. and Hirao, Y., Prediction Traffic Control for MAGLEV System and Its Effect, (in Japanese), 1984 IEEJ Conference, No.772 (1984), pp.948-949.

( 3 ) Hiraguri, S., Hirao, Y., Watanabe, I., Tomii, N. and Hase, S., Train Control Method Based on Prediction of Traffic, (in Japanese), Proceedings of IEEJ Transportation and Electric Railway Conference, TER-99-70, (1999), pp.25-30.

( 4 ) Watanabe, I., Fukuda, M., Ushijima, Y. and Takashige, T., Development of Digital ATC System, Quarterly Report of RTRI, Vol.40, No.1 (1999), pp.32-36.

( 5 ) Technical Committee of Transportation and Electric Railway, IEEJ: Transport and Energy, (in Japanese), IEEJ Technical Report, No.597 (1996), p.24.

(6) Hasegawa, Y., Development Steps in Application of CARAT, Quarterly Report of RTRI, Vol.36, No.1 (1995), pp.4-7. 